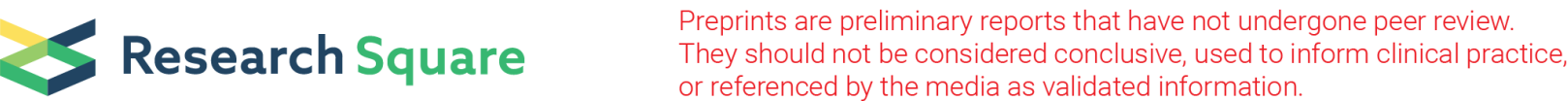

\section{Efficacy of Different Nucleoside Analog Rescue Therapies for Entecavir-Resistant Chronic Hepatitis B Patients}

\section{Jin Shang}

West China Hospital, Sichuan University

Juan Zhou

Sichuan University West China Hospital

Huan Liu

Sichuan University West China Hospital

You Tu

Sichuan University West China Hospital

Jinqiu Ran

Sichuan University West China Hospital

Lang Bai ( $\nabla$ pangbailang@163.com )

West China Hospital, Sichuan University

Hong Tang

Sichuan University West China Hospital

\section{Research article}

Keywords: Hepatitis B virus, Chronic hepatitis B, Antiviral treatment, Entecavir, Tenofovir, Adefovir, Drug resistance, Rescue therapy, Virologic response, Renal safety

Posted Date: August 31st, 2020

DOI: https://doi.org/10.21203/rs.3.rs-64765/v1

License: (c) (1) This work is licensed under a Creative Commons Attribution 4.0 International License. Read Full License 


\section{Abstract}

Background: Entecavir (ETV) is recommended as a first-line anti-HBV treatment. However, many chronic hepatitis B patients initiate anti-HBV treatment such as lamivudine and telbivudine with low genetic barriers in China, which leads to compensatory mutations and increases the rate of ETV resistance. The management of ETV resistance in China is an essential clinical issue.

Methods: Patients from 2011 to 2017 with nucleos(t)ide analog resistance were screened and 72 patients with ETV resistance were included. These patients received different rescue therapies including an ETV and adefovir (ADV) combination therapy group $(n=25)$, a tenofovir (TDF) monotherapy group $(n=27)$, and an ETV and TDF combination therapy group $(n=20)$. Virologic, biochemical, and serologic responses were compared among the three groups.

Results: The rate of ETV resistance increased form $6.04 \%$ in 2011 to $15.02 \%$ in 2017. Regarding the rates of negative HBV DNA at 48 weeks, no significant differences occurred in the TDF monotherapy and TDF combination groups (74.07\% vs $70.00 \%)$, while the ETV and ADV group showed the worst virologic response (28.00\%). TDF monotherapy and TDF combination therapy showed similar decline of HBV DNA at weeks 12,24 , and 48 . There was no significant difference in the rates of HBeAg clearance, ALT normalization, and abnormal renal function between the three groups.

Conclusions: TDF monotherapy showed a comparable virologic response to TDF and ETV combination therapy and a better virologic response than ETV and ADV combination therapy. Thus, TDF monotherapy is the preferred rescue therapy for ETV resistance.

\section{Introduction}

The World Health Organization estimates that 257 million people around the world are currently infected with hepatitis B virus (HBV) and approximately 63 million new cases will occur between 2015 and 2030 [1]. Management of HBV infection remains a global public health challenge. At present, curing HBV is challenging in most patients and they need long-term antiviral treatment. Entecavir (ETV) is recommended as the first-line antiviral treatment in the APASL, AASLD, and EASL guidelines [2-4], and the drug resistance of ETV is only $1 \%$ over 5 years in treatment-naive patients.

It is reported that approximately $81 \%$ of chronic hepatitis $\mathrm{B}(\mathrm{CHB})$ patients receive antiviral drugs with low barrier to resistance such as lamivudine (LMV) and telbivudine (LDT) or adefovir (ADV) before ETV treatment in China [5]. If primary resistance mutations occur, compensatory mutations may arise even if primary treatment is stopped. The rate of ETV resistance increases to $51 \%$ in LMV-resistant patients [6]. Drug-resistant patients have higher rates of hepatitis flares and disease progression [7]. Therefore, management of ETV resistance has become an essential clinical issue in China.

Among rescue therapies for patients with ETV resistance, according to the APASL, AASLD, and EASL guidelines $[2,3,4]$, for patients with only ETV resistance, switching to TDF is recommended, while in 
patients with multi-drug resistance, TDF or a combination of ETV and TDF are recommended. Because TDF has a high barrier to drug resistance [8], both TDF monotherapy and TDF combined with ETV showed high virologic responses in patients with ETV resistance [9]. Moreover, HBV variants of ADV resistance are also not cross-resistant to ETV, so ETV and ADV combination therapy could be considered in theory, and data in a recent report supported this view [10]. Combining ETV and ADV is also recommended in the APASL guidelines. However, the AASLD and EASL guidelines do not recommended combining ETV and ADV as ETV-resistant rescue therapy. Many patients receive ADV treatment in China because of its relatively low cost. ETV and ADV combination therapy is an alternative rescue therapy for ETV-resistant patients according to the Chinese CHB guidelines by the Chinese Society of Infectious Diseases and Chinese Society of Hepatology. Due to the lack of data on comparative research into ETVADV combination therapies to TDF monotherapy or TDF-ETV combination therapy, whether combination ETV and ADV therapy has a comparable efficacy with TDF or TDF plus ETV is worth evaluating.

Therefore, this study compared the efficacy of ETV plus ADV combination therapy, TDF monotherapy, and ETV and TDF combination therapy in ETV-resistant patients.

\section{Patients And Methods}

The study protocol was approved by the ethics committee of West China Hospital at Sichuan University. Patients were included from the Center of Infectious Diseases, West China Hospital, from 2011 to 2017. Patients with persistent HBV viremia or virologic breakthrough were eligible for enrollment at screening. Patients with confirmed ETV genotypic resistance mutations were included.

Patients with underlying liver diseases such as viral hepatitis, nonalcoholic fatty liver diseases, and autoimmune hepatitis were excluded. Patients with underlying severe chronic respiratory diseases, cardiovascular disease, and chronic kidney injury were not enrolled. Patients lost to complete follow-up were also excluded. Ultimately, 72 patients with ETV resistance (rtL180M, rtT184A/C/F/G/I/L/S, rtS202G, rtM250L/V, and rtM204V/I) combined with ADV resistance (rtA181V/T and/or rtN236T) were included. The patients switched to three rescue therapies, including ETV/ADV combination therapy, TDF monotherapy, and ETV/TDF combination therapy. After 48 weeks of rescue treatment in patients with ETV resistance, the virologic response, rate of normal alanine aminotransferase (ALT), and incidence of HBeAg loss/seroconversion were compared in the three groups. The HBV DNA levels at 12 weeks, 24 weeks, and 48 weeks were recorded. Adverse events were assessed throughout 48 weeks. The glomerular filtration rate was estimated using the modification of diet in renal disease equation as follows: estimated glomerular filtration rate (eGFR; milliliters per minute per $1.73 \mathrm{~m}^{2}$ ) $=186$ * serum creatinine ${ }^{-}$ $1.154 *$ age $^{-0.203 *}(0.742$ if female) * 1.233 (Chinese). (Supplementary Fig. 1)

\section{Statistical analysis}

Group-group comparisons of continuous variables were conducted using the analysis of variance, $t$ test, chi-squared test, or Fisher's exact test. All of the statistical analyses were conducted using SPSS version 22.0. $\mathrm{P}<0.05$ was considered statistically significant. 


\section{Results}

\section{Trends in ETV resistance mutations}

The rates of ETV resistance gradually increased from $6.04 \%$ in 2011 to $15.02 \%$ in 2017 , and the proportion of LAM/LdT resistance was high from 2011 to 2017. Overall, 166 patients with ETV resistance were screened and 72 were included (Fig. 1 and Supplementary Fig. 1).

\section{Baseline characteristics of patients with ETV resistance}

The 72 patients in the ETV/ADV $(n=25), \operatorname{TDF}(n=27)$, and ETV/TDF groups $(n=20)$ completed 48 weeks of treatment. The median age was 43,44 , and 46 years in the ETV/ADV, TDF, and ETV/TDF groups, respectively. The proportion of males was $80.00 \%, 85.19 \%$, and $65.00 \%$ in the ETV/ADV, TDF, and ETV/TDF groups, respectively. The median HBV DNA level was $6.31,5.40$, and $6.23 \log _{10} \mathrm{lU} / \mathrm{mL}$, respectively. No significant differences occurred in the age distribution, male proportion, baseline HBV DNA level or other liver function, and renal function tests (Table 1). 
Table 1

Baseline characteristics of patients with ETV-resistance in different rescue therapy groups

\begin{tabular}{|llll|}
\hline & ETV + ADV & TDF & TDF + ETV \\
\hline $\mathrm{N}$ & 25 & 27 & 20 \\
\hline Age, years & $43(21-64)$ & $44(20-64)$ & $46(30-68)$ \\
\hline Male, $\mathrm{n}(\%)$ & $20,80.00 \%$ & $23,85.19 \%$ & $13,65 \%$ \\
\hline ALT (IU/L) & 120.46 & 61.12 & 71.00 \\
\hline Normal ALT, $\mathrm{n}(\%)$ & $13,52.00 \%$ & $17,62.96 \%$ & $10,50.00 \%$ \\
\hline Bilirubin, mg/dL & 18.93 & 20.67 & 33.86 \\
\hline Creatinine, mg/dL & 78.97 & 90.62 & 75.43 \\
\hline HBeAg-positivity, $\mathrm{n}(\%)$ & $17,67.00 \%$ & $23,85.19 \%$ & $15,75.00 \%$ \\
\hline HBV DNA, log10 IU/mL & 6.31 & 5.40 & 6.23 \\
\hline Previously exposed NUC, $\mathrm{n}$ & & & \\
\hline LMV & 23 & 14 & 14 \\
\hline ADV & 0 & 6 & 3 \\
\hline LDT & 1 & 2 & 1 \\
\hline LMV + ADV & 1 & 5 & 2 \\
\hline Resistance mutations & & 5 & 17 \\
\hline ETV & 18 & 22 & 3 \\
\hline Multidrug resistance & 7 & 5 & \\
\hline
\end{tabular}

\section{Virologic, biochemical, and serologic responses in the subgroups}

Three patients in the ETV/ADV group discontinued because of poor virologic response and switched to ETV/TDF combination therapy. One patient in the TDF group discontinued because of risk of renal injury. Among the patients in the TDF and ETV/TDF groups, no significant difference was found in the rate of achieving virologic response at 48 weeks (74.07\% vs $70.00 \%)$. The mean level of HBV DNA significantly decreased at 12 weeks, 24 weeks, and 48 weeks, and no virologic breakthrough occurred in these two groups. However, the ETV/ADV group showed a significantly low rate of achieving virologic response at 48 weeks (28\%). The residual HBV DNA level was also significantly higher than in the TDF or ETV/TDF groups. Virologic breakthrough occurred in one patient after 40 weeks of ETV/ADV treatment. The patient 
switched to ETV/TDF, and their HBV DNA declined to negative after 12 weeks of treatment. As for serologic responses, HBeAg seroclearance occurred in 3 patients in the TDF group, 1 patient in the ETV/TDF group, and 0 patients in the ETV/ADV group, with no significant difference (Table 2 and Fig. 2). To evaluate whether poor virologic response in the ETV/ADV group was due to the relatively higher rate of multi-drug resistance, we conducted a subgroup analysis of ETV resistance without ADV resistance. We found that in the patients with only ETV resistance, the ETV/ADV group still demonstrated a significantly lower rate of virologic response at 48 weeks, and the residual HBV DNA level was higher than in the other two groups (Table 3).

Table 2

Virologic, biochemical, and serologic responses of ETV-resistance patients with/or without ADV resistance in different rescue therapy at Week 48

\begin{tabular}{|llll|}
\hline & ETV + ADV & TDF & TDF + ETV \\
\hline $\mathrm{N}$ & 25 & 27 & 20 \\
\hline HBV DNA < 100 IU/mL, $\mathrm{n}(\%)$ & $7,28.00 \%$ & $20,74.07 \%$ & $14,70 \%$ \\
\hline HBV DNA change from baseline $(\log 10 \mathrm{IU} / \mathrm{mL})$ & $3.86 \pm 1.62$ & $4.55 \pm 1.81$ & $5.33 \pm 1.88$ \\
\hline Residual HBV DNA level $(\log 10 \mathrm{IU} / \mathrm{mL})$ & 2.44 & 0.86 & 0.8 \\
\hline Virologic breakthrough & 1 & 0 & 0 \\
\hline ALT $(\mathrm{IU} / \mathrm{L})$ & 40.56 & 45.33 & 34.82 \\
\hline ALT normal, $\mathrm{n}(\%)$ & $19,76.00 \%$ & $23,85.19 \%$ & $16,80.00 \%$ \\
\hline Abnormal renal function & 0 & 1 & 1 \\
\hline HBeAg seroclearance, $\mathrm{n}(\%)$ & 0 & 3 & 1 \\
\hline HBeAg seroconversion, $\mathrm{n}(\%)$ & 0 & 1 & 1 \\
\hline HBsAg seroclearance, $\mathrm{n}(\%)$ & 0 & 0 & 0 \\
\hline Discontinued & 3 & 1 & 0 \\
\hline
\end{tabular}


Table 3

Subgroup analysis of virologic, biochemical, and serologic responses of patients with only ETV resistance different rescue therapy at Week 48

\begin{tabular}{|llll|}
\hline & ETV + ADV & TDF & TDF + ETV \\
\hline $\mathrm{N}$ & 18 & 22 & 17 \\
\hline HBV DNA < 100 IU/mL, $\mathrm{n}(\%)$ & $5,27.28 \%$ & $15,68.18 \%$ & $11,64.71 \%$ \\
\hline HBV DNA change from baseline $(\log 10 \mathrm{IU} / \mathrm{mL})$ & 4.09 & 4.07 & 5.03 \\
\hline Residual HBV DNA level (log10 IU/mL) & 2.45 & 1.19 & 1.03 \\
\hline Virologic breakthrough & 0 & 0 & 0 \\
\hline ALT (IU/L) & 41.44 & 44.1 & 35.13 \\
\hline ALT normal, $\mathrm{n}(\%)$ & $13,72.22 \%$ & $19,86.36 \%$ & $14,82.35 \%$ \\
\hline Abnormal renal function & 0 & 0 & 1 \\
\hline HBeAg seroclearance, $\mathrm{n}(\%)$ & 0 & $3,13.64 \%$ & $1,5.88 \%$ \\
\hline HBeAg seroconversion, $\mathrm{n}(\%)$ & 0 & $1,4.55 \%$ & $1,5.88 \%$ \\
\hline HBsAg seroclearance, $\mathrm{n}(\%)$ & 0 & 0 & 0 \\
\hline Discontinued & 3 & 1 & 0 \\
\hline 12 weeks HBV DNA level $(\log 10 \mathrm{IU} / \mathrm{mL})$ & 3.75 & 2.47 & 2.83 \\
\hline 24 weeks HBV DNA level $(\log 10 \mathrm{IU} / \mathrm{mL})$ & 2.44 & 1.36 & 1.84 \\
\hline 48 weeks HBV DNA level $(\log 10 \mathrm{IU} / \mathrm{mL})$ & 2.45 & 1.19 & 1.03 \\
\hline 12 weeks HBV DNA < 100 IU/mL, $\mathrm{n}(\%)$ & $0.00 \%$ & $27.27 \%$ & $17.65 \%$ \\
\hline 24 weeks HBV DNA < 100 IU/mL, $\mathrm{n}(\%)$ & $16.67 \%$ & $54.55 \%$ & $41.18 \%$ \\
\hline 48 weeks HBV DNA < 100 IU/mL, $\mathrm{n}(\%)$ & $27.78 \%$ & $68.18 \%$ & $64.71 \%$ \\
\hline
\end{tabular}

\section{Safety profiles}

Regarding the safety of the different treatments, only 1 hepatocellular carcinoma occurred in the ETV/ADV combination group, and no ALT flares occurred in either group. No significant difference occurred in the discontinuation rate due to adverse events in the three groups. As for eGFR measurement, differences in the baseline eGFR level, eGFR at 48 weeks, and eGFR decline from baseline or the rate of eGFR $<90 \mathrm{~mL} / \mathrm{min} / 1.73 \mathrm{~m} 2$ at 48 weeks demonstrated no significant differences (Table 4 and Supplementary Figure 2). 


\section{Safety profiles}

Regarding the safety of the different treatments, only 1 hepatocellular carcinoma occurred in the ETV/ADV combination group, and no ALT flares occurred in either group. No significant difference occurred in the discontinuation rate due to adverse events in the three groups. As for eGFR measurement, differences in the baseline eGFR level, eGFR at 48 weeks, and eGFR decline from baseline or the rate of eGFR $<90 \mathrm{~mL} / \mathrm{min} / 1.73 \mathrm{~m} 2$ at 48 weeks demonstrated no significant differences (Table 4 and Supplementary Figure 2).

Table 4

Safety Profiles of patients in different rescue therapy group at 48 weeks

\begin{tabular}{|llll|}
\hline & ETV+ADV & TDF & TDF+ETV \\
\hline N & 25 & 27 & 20 \\
\hline cirrhosis & 1 & 0 & 0 \\
\hline Deaths & 0 & 0 & 0 \\
\hline ALT flare & 0 & 0 & 0 \\
\hline Discontinuation due to adverse event & 2 & 0 & 0 \\
\hline baseline eGFR & 0 & 1 & 1 \\
\hline eGFR at 48 weeks & 125.82 & 122.75 & 127.71 \\
\hline eGFR decline from baseline & 103.76 & 112.32 & 110.26 \\
\hline eGFR $<90 \mathrm{~mL} / \mathrm{min} / 1.73 \mathrm{~m}^{2}$ at 48 weeks, $\mathrm{n}(\%)$ & $3,12.00 \%$ & $1,3.70 \%$ & $3,15.00 \%$ \\
\hline
\end{tabular}

\section{Discussion}

From 2011 to 2017, the rate of ETV resistance among all HBV-resistant variants increased from 6.04$15.02 \%$. ETV resistance is becoming severe in China because antiviral drugs such as LMV, LDT, and ADV with low barriers to resistance are commonly used, and the number of $\mathrm{CHB}$ patients constitutes approximately one-half of the global CHB population [11]. Many treatment-experienced CHB patients have a higher risk of ETV resistance. As for the mechanism of ETV resistance, the primary mutations of LMV resistance are the methionine residues at amino acid 204 conferred to isoleucine or valine M204 I/V, while methionine or serine changes at $180(\mathrm{rtL} 180 \mathrm{M} / \mathrm{S})$ usually accompany this [12]. Once such primary resistance mutation occurred, the ETV resistance, which needs a mutation at B domain (rt1169T or rtS184G), C domain (rtS202G/l), or E domain (rtM250V) on basis of rtM204V/I \pm rtL180M/S mutation, is much more likely to achieve [13]. Therefore, monitoring the resistance of ETV requires more attention. 
Regarding the efficacy of different rescue therapies for patients with ETV resistance, in our study, TDF and TDF plus ETV combination therapy showed comparable virologic response at 24 or 48 weeks, which was similar to previous studies on patients with partial virologic response to ETV $[14,15]$. Even in multidrug-resistant CHB patients, TDF demonstrated comparable efficacy and safety to TDF plus ETV combination therapy [16]. Theoretically, N236T and A194T mutations are potential variants with resistance to TDF, which has no cross-resistance to ETV. Because ETV has minimal antiviral effects in patients with ETV resistance, TDF monotherapy is more likely to have comparable antiviral effects compared with TDF plus ETV combination therapy. However, one major concern is that TDF genetic resistance occurred in LMV-experienced patients [17], and an rtL180M/T184L/A200V/M204V mutation with resistance to TDF was found in ETV-resistant patients receiving TDF monotherapy [18]. Whether primary resistant mutations to ETV resistance could increase the rate of mutations resistant to TDF in long-term TDF monotherapy is unknown. However, long-term clinical trials on TDF and TDF plus ETV combination therapy demonstrated that no persistent HBV viremia or virologic breakthroughs occurred in TDF monotherapy at 144 weeks or 240 weeks $[16,19]$. In one in vitro study, TDF was susceptible in both LMV-resistant clones and ETV-resistant clones [20]. Therefore, we posit that in ETV-resistant patients, TDF monotherapy has a low rate of secondary mutations resistant to TDF. Whether ETV plus ADV resistance increases the potential for TDF resistance requires long-term follow-up studies for clarification.

Regarding the safety profile of the TDF and TDF plus ETV groups, the baseline eGFR and eGFR at 48 weeks were comparable between these two groups. TDF is excreted via glomerular filtration, and active tubular transport and may cause proximal tubular dysfunction [21]. Proximal tubular dysfunction was not found in ETV treatment. However, there was no evidence that a combination of ETV and TDF could reduce the potential for TDF renal toxicity $[22,23]$. Therefore, we posit that no difference in renal safety exists between TDF monotherapy and TDF plus ETV combination therapy. Because long-term adherence and the cost-effectiveness of monotherapy is better than combination therapy, TDF monotherapy could be optimal treatment strategy for patients with underlying renal or bone metabolism diseases.

The virologic response of ETV plus ADV combination therapy is the worst among the three therapies regardless of multi-drug resistance. Only $28 \%$ of patients achieved virologic response, and $72 \%$ of patients had persistent HBV viremia at 48 weeks, demonstrating a higher occurrence rate of cirrhosis or hepatocellular carcinoma in long-term treatment. Although major ADV-resistant mutations were rtA181V/T mutations and rtN235T without cross-resistance to ETV, the relatively weak antiviral efficacy of ADV limits its use in ETV-resistant patients. In previous research, ADV had an estimated 30\% resistance rate after 5 years of treatment in LMV-resistant patients [24]. Moreover, ADV and LMV dual resistant mutations may occur in LMV-resistant patients receiving ADV rescue therapy [25]. Additionally, TDF is easy to access and extremely inexpensive because of the new government procurement policy in China. ETV and ADV combination therapy has lower antiviral efficacy without the advantage of safety or cost-effectiveness. Therefore, TDF monotherapy may be the preferred rescue therapy rather than ETV and ADV combination therapy. 


\section{Conclusion}

ETV resistance has gradually become a severe clinical problem in China because of the large number of antiviral treatment-experienced patients. TDF showed comparable virologic response and tolerance to TDF plus ETV combination therapy and better virologic response than ETV plus ETV combination therapy. TDF monotherapy may be the optimal strategy for CHB patients with ETV resistance.

\section{Declarations}

\section{Ethics approval and consent to participate}

The study protocol was approved by the ethics committee of West China Hospital at Sichuan University. All including patients from the Center of Infectious Diseases, West China Hospital signed informed consents.

\section{Consent for publication}

The submitted manuscripts have not been published elsewhere and are not currently under consideration for publication by another journal.

\section{Competing interests}

The authors declare they have no conflicts of interest.

\section{Funding}

This study was supported by grants from the National Science and Technology Major Project of China 2017ZX10202202-002-004 and the Science and Technology Project of Chengdu (2017-CY02-00018-GX).

\section{Authors' contributions}

Jin Shang and Juan Zhou were equally contributed to data collection, analysis and manuscript writing, Huan Liu, Rili M ise, You Tu, Jinqiu Ran were contributed to follow-up of patients and data collection, Lang Bai and Hong Tang were equally contributed to design of the study and writing help, monitoring of the study process.

\section{Acknowledgements}

None. 


\section{References}

1. Nayagam S, Thursz M, Sicuri E, et al. Requirements for global elimination of hepatitis B: a modelling study. Lancet Infect Dis.2016;16:1399-1408.

2. Sarin SK, Kumar M, Lau GK, et al. Asian-Pacific clinical practice guidelines on the management of hepatitis B: a 2015 update. Hepatol Int. 2016;10(1):1-98.

3. Terrault NA, Lok ASF, McMahon BJ, et al. Update on Prevention, Diagnosis, and Treatment of Chronic Hepatitis B: AASLD 2018 Hepatitis B Guidance. Clin Liver Dis (Hoboken). 2018;12(1):33-34.

4. European Association for the Study of the Liver. European Association for the Study of the Liver. EASL 2017 Clinical Practice Guidelines on the management of hepatitis B virus infection. J Hepatol. 2017;67(2):370-398.

5. Zhuang $\mathrm{H}$, Weng $X$. Development and management of drug resistance to nucle- oside/nucleotide analogues in patients with chronic hepatitis B. Chin J Hepatol 2013;21(1):15-22 .

6. Tenney DJ, Rose RE, Baldick CJ, et al. Two-year assessment of entecavir resistance in Lamivudinerefractory hepatitis $B$ virus patients reveals different clinical outcomes depending on the resistance substitutions present. Antimicrob Agents Chemother 2007;51:902-911.

7. Yuen MF, Seto WK, Chow DH, et al. Long-term lamivudine therapy reduces the risk of long-term complications of chronic hepatitis B infection even in patients without advanced disease. Antivir Ther 2007;12:1295-1303.

8. Liu Y, Miller MD, Kitrinos KM. Tenofovir alafenamide demonstrates broad cross-genotype activity against wild-type HBV clinical isolates and maintains susceptibility to drug-resistant HBV isolates in vitro. Antiviral Res 2017;139:25-31.

9. Zoulim F, Jablkowski MS, Diculescu M, et al. The safety and efficacy of entecavir and tenofovir combination therapy for chronic hepatitis $B$ in patients with previous nucleos(t)ide treatment failure: week 96 results of the ENTEBE study. Hepatology 2014;60(Suppl 4):314A-315A

10. Kim HS, Yim HJ, Jang MK, et al. Management of entecavir-resistant chronic hepatitis B with adefovirbased combination therapies. World J Gastroenterol. 2015;21(38):10874-10882.

11. Wang FS , Fan JG , Zhang Z , Gao B , Wang HY. The global burden of liver disease: the major impact of China. Hepatology 2014;60:2099-108 .

12. Tong S, Revill P. Overview of hepatitis B viral replication and genetic variability. J Hepatol 2016;64:S416.

13. Zoulim F, Locarnini S. Optimal management of chronic hepatitis B patients with treatment failure and antiviral drug resistance. Liver Int 2013;33 Suppl 1:116-24.

14. Lu L, Yip B, Trinh H, et al. Tenofovir-based alternate therapies for chronic hepatitis B patients with partial virological response to entecavir. J Viral Hepat. 2015;22(8):675-681.

15. Chen J, Zhao SS, Liu XX, Huang ZB, Huang Y. Comparison of the Efficacy of Tenofovir Versus Tenofovir plus Entecavir in the Treatment of Chronic Hepatitis B in Patients With Poor Efficacy of Entecavir: A Systematic Review and Meta-analysis. Clin Ther. 2017;39(9):1870-1880. 
16. Lim YS, Gwak GY, Choi J, et al. Monotherapy with tenofovir disoproxil fumarate for adefovir-resistant vs. entecavir-resistant chronic hepatitis B: A 5-year clinical trial. J Hepatol. 2019;71(1):35-44.

17. Alacam S, Karabulut N, Yolcu A, et al. Evaluation of drug resistance mutations in patients with chronic hepatitis B. Folia Microbiol (Praha). 2019;64(2):237-243.

18. Jiang D, Wang J, Zhao X, et al. Entecavir resistance mutations rtL180M/T184L/M204V combined with rtA200V lead to tenofovir resistance. Liver Int. 2020;40(1):83-91.

19. Lim YS, Lee YS, Gwak GY, et al. Monotherapy with tenofovir disoproxil fumarate for multiple drugresistant chronic hepatitis B: 3-year trial. Hepatology. 2017;66(3):772-783.

20. Murakami E, Tsuge M, Hiraga N, et al. Effect of tenofovir disoproxil fumarate on drug-resistant HBV clones. J Infect. 2016;72(1):91-102.

21. Herlitz LC, Mohan S, Stokes MB, Radhakrishnan J, D'Agati VD, Markowitz GS. Tenofovir nephrotoxicity: acute tubular necrosis with distinctive clinical, pathological, and mitochondrial abnormalities. Kidney Int. 2010;78(11):1171-1177.

22. Lee YB, Lee JH, Lee $\mathrm{DH}$, et al. Efficacy of entecavir-tenofovir combination therapy for chronic hepatitis B patients with multidrug-resistant strains. Antimicrob Agents Chemother. 2014;58(11):6710-6716.

23. Lim YS, Byun KS, Yoo BC, et al. Tenofovir monotherapy versus tenofovir and entecavir combination therapy in patients with entecavir-resistant chronic hepatitis B with multiple drug failure: results of a randomised trial. Gut. 2016;65(5):852-860.

24. Salpini R, Alteri C, Cento V, et al. Snapshot on drugresistance rate and profiles in patients with chronic hepatitis B receiving nucleos(t)ide analogues in clinical practice. J Med Virol 2013;85:996-1004.

25. Fung SK, Andreone P, Han SH, et al. Adefovir-resistant hepatitis B can be associated with viral rebound and hepatic decompensation. J Hepatol 2005;43:937-43.

\section{Figures}




\section{A}

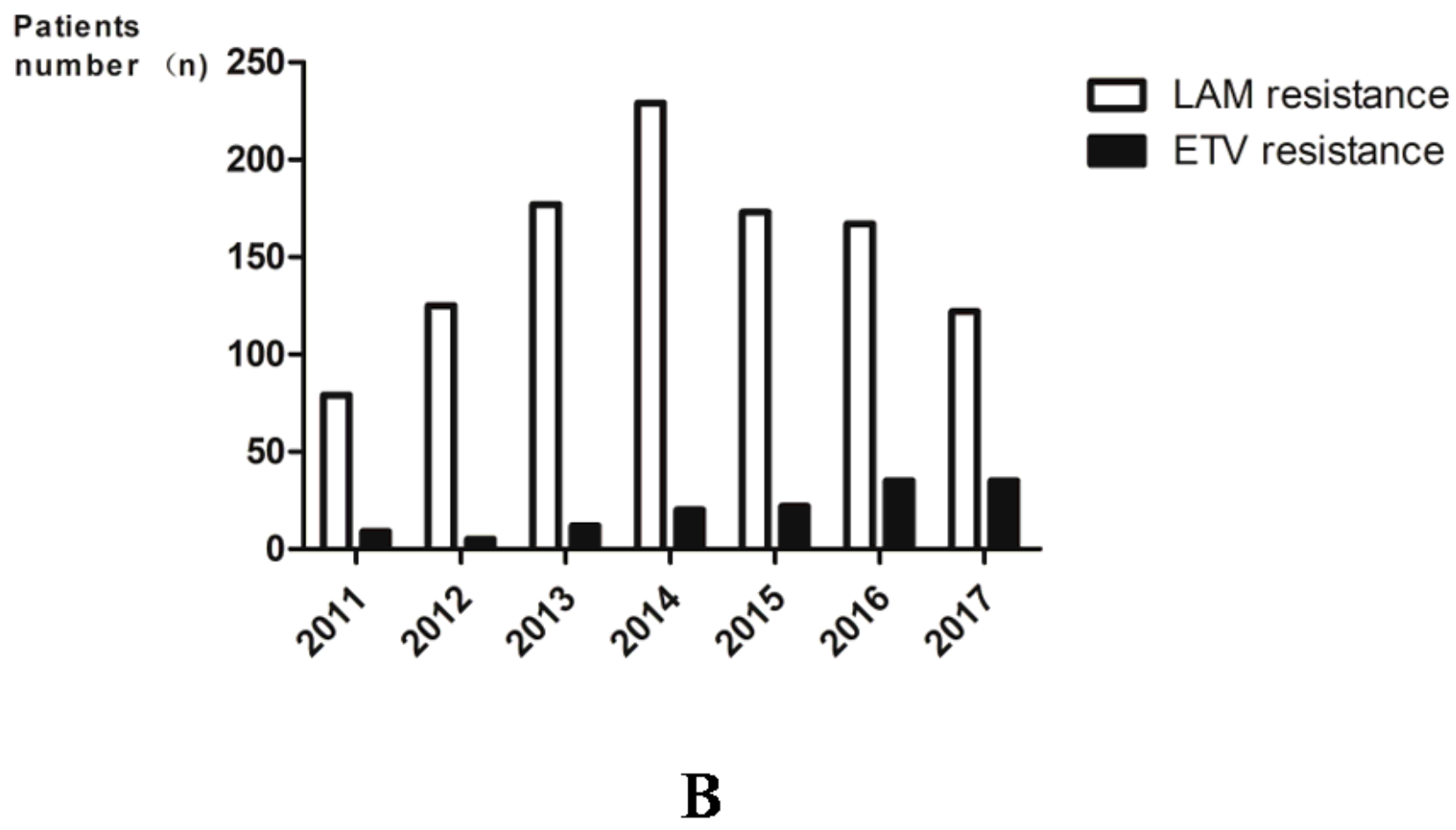

Percentage(100\%)

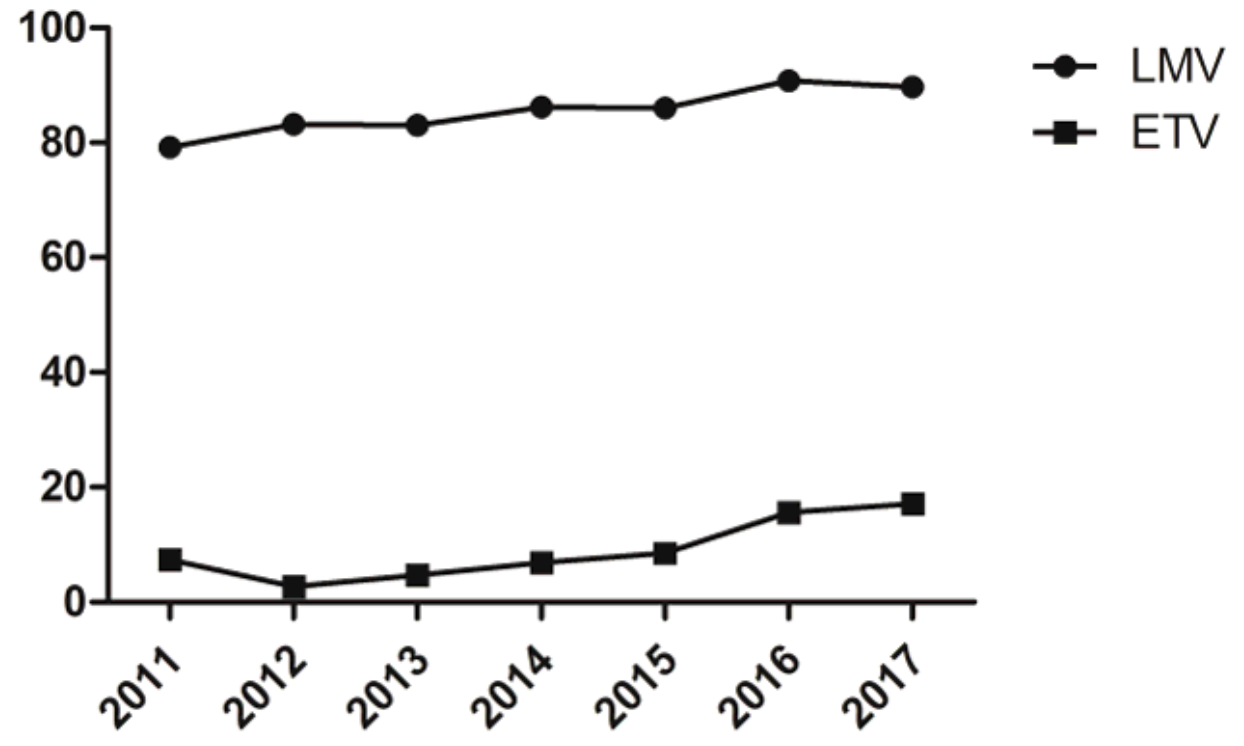

Figure 1

Trends of nucleos(t)ide analogue resistance mutations Occurrence rate of ETV resistance varies from 2011-2017, (A) Number of patients with LMV resistance and ETV resistance; (B) Proportion of LMV resistance and ETV resistance among all patients with nucleos(t)ide analogue resistance. 
A
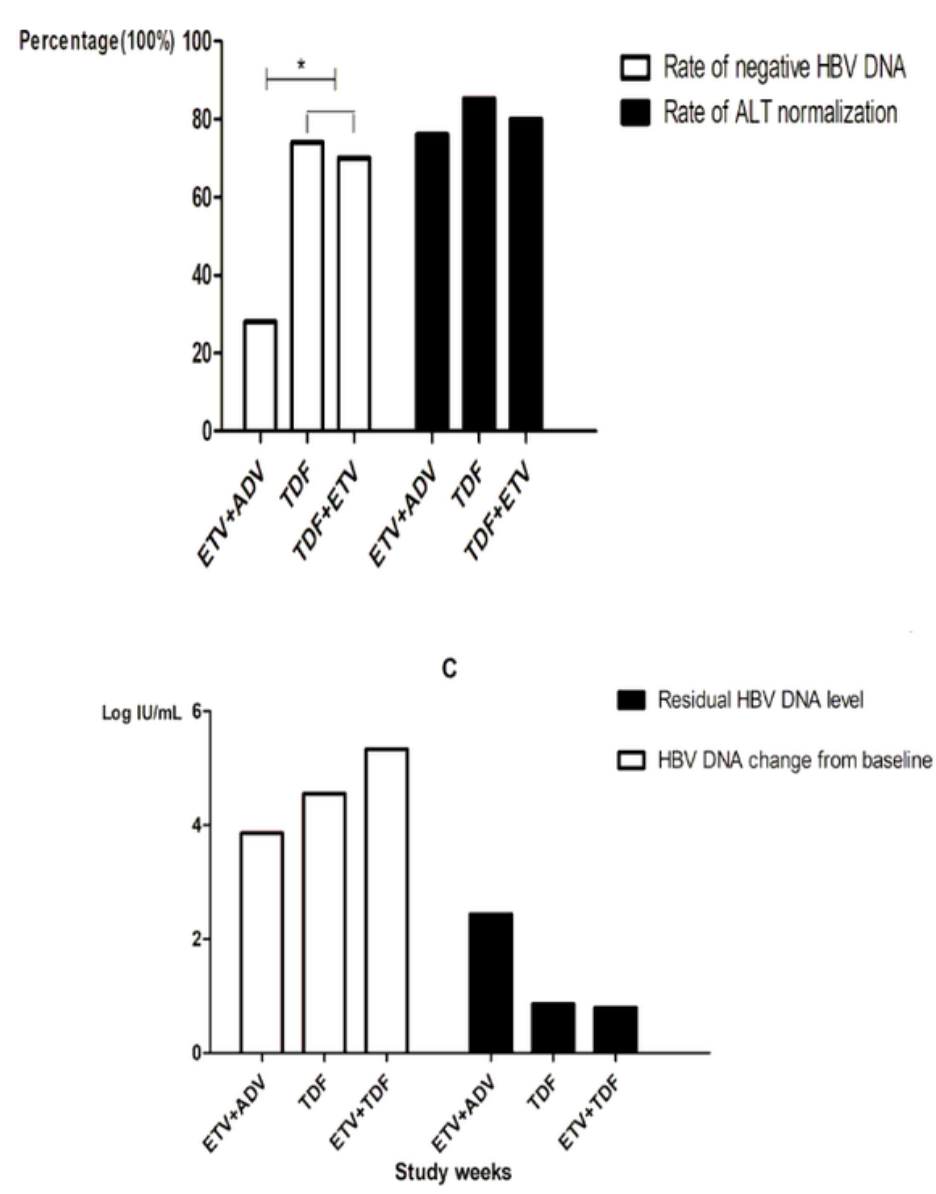

B
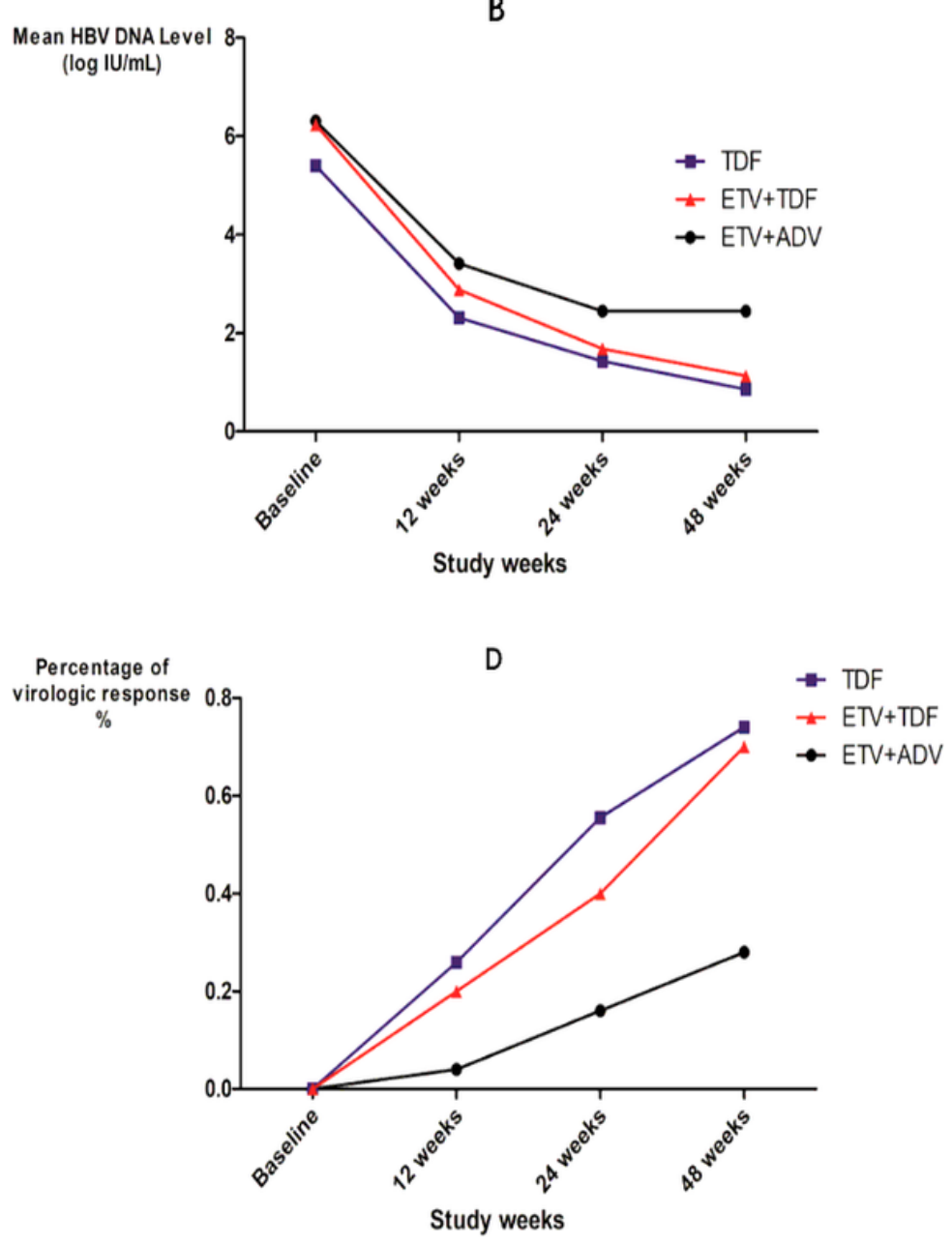

\section{Figure 2}

Virologic, biochemical, and serologic responses of different rescue therapy Virologic response, biochemical response and serologic response in three groups were analyzed, (A) Rate of negative HBV DNA and rate of ALT normalization in different rescue therapy groups. (B) Mean HBV DNA level decline at 12, 24, 48 weeks in different rescue therapy groups. (C) Residual HBV DNA and HBV DNA change from baseline level in different rescue therapy groups. (D) Percentage of virologic response in different rescue therapy groups.

\section{Supplementary Files}

This is a list of supplementary files associated with this preprint. Click to download.

- SupplementaryFigure2.tif

- SupplementaryFlgure1.tif 